\title{
The initial value problem in numerical relativity
}

\author{
Harald P. Pfeiffer \\ Theoretical Astrophysics, California Institute of Technology, Pasadena, California 91125 \\ Received (Day Mth. Year) \\ Revised (Day Mth. Year) \\ Communicated by [editor]
}

\begin{abstract}
The conformal method for constructing initial data for Einstein's equations is presented in both the Hamiltonian and Lagrangian picture (extrinsic curvature decomposition and conformal thin sandwich formalism, respectively), and advantages due to the recent introduction of a weight-function in the extrinsic curvature decomposition are discussed. I then describe recent progress in numerical techniques to solve the resulting elliptic equations, and explore innovative approaches toward the construction of astrophysically realistic initial data for binary black hole simulations.
\end{abstract}

Keywords: Einsteins equations; initial value problem; numerical relativity.

\section{Introduction}

Numerical methods play an important role for investigations into the properties of Einstein's equations. In particular, the late stages of inspiral and coalescence of binary compact objects like binary black holes are thought to be accessible only to numerical investigations. Knowledge of the full waveform of inspiraling binary black holes, including the highly nonlinear coalescence phase, will enhance sensitivity of gravitational wave detectors like LIGO or GEO600 through cross-correlation of the observed signal with the expected waveforms [20]. Comparison of the observed signals with the predictions of general relativity will test general relativity in the genuinely nonlinear regime. Besides the experimental urgency, the binary black hole problem is arguably the most fundamental dynamical problem in general relativity; however, it remains unsolved.

Initial data forms the starting point for any evolution. For Einstein's equations, the most widely used method to construct initial data is the conformal method, pioneered by Lichnerowicz [30] and extended to a more general form by York and coworkers 49 3452]. In two recent papers, York [53] and Pfeiffer \& York 40] completed the conformal method: It is now available in a Lagrangian and in a Hamiltonian picture (referred to as the conformal thin sandwich formalism and the extrinsic curvature decomposition, respectively), and both pictures completely agree. The transverse-tracefree part of the extrinsic curvature is now defined such that it 
vanishes for any stationary spacetime. The method is now completely invariant to conformal transformations of the free data.

The conformal method results in a set of coupled nonlinear three-dimensional elliptic partial differential equations. Over the last few years, numerical techniques for solving these coupled elliptic equations were improved tremendously. Construction of binary black hole initial data is no longer limited by numerical capabilities, but by the incomplete understanding of the choice of free data and boundary conditions for the elliptic equations.

Here, we present the Lagrangian and Hamiltonian pictures of the conformal method, including many details which may have been mentioned in passing in technical papers, but were never presented in a coherent fashion. In the second part of this paper, we describe briefly numerical methods and then explore recent innovative approaches to the construction of astrophysically realistic binary black hole initial data. Throughout this paper, emphasis is placed on physical and numerical issues, rather than mathematical proofs.

\section{The initial value problem}

Using the standard 3+1 decomposition [152 of Einstein's equations, we foliate spacetime with spacelike $t=$ const. hypersurfaces. Each such hypersurface has a future pointing unit-normal $n^{\mu}$, induced metric $g_{\mu \nu}={ }^{(4)} \mathbf{g}_{\mu \nu}+n_{\mu} n_{\nu}$ and extrinsic curvature $K_{\mu \nu}=-\frac{1}{2} \mathcal{L}_{n} g_{\mu \nu}$. The spacetime metric can be written as

$$
d s^{2}=-N^{2} d t+g_{i j}\left(d x^{i}+\beta^{i} d t\right)\left(d x^{j}+\beta^{j} d t\right),
$$

where $N$ and $\beta^{i}$ denote the lapse function and shift vector, respectively. $N$ measures the proper separation between neighboring hypersurfaces along the surface normals and $\beta^{i}$ determines how the coordinate labels move between hypersurfaces: Points along the integral curves of the time-vector $t^{\mu}=N n^{\mu}+\beta^{\mu}$ (where $\beta^{\mu}=\left[0, \beta^{i}\right]$ ), have the same spatial coordinates $x^{i}$.

Einstein's equations decompose into evolution equations and constraint equations for the quantities $g_{i j}$ and $K_{i j}$. The evolution equations determine how $g_{i j}$ and $K_{i j}$ are related between neighboring hypersurfaces,

$$
\begin{aligned}
\partial_{t} g_{i j}= & -2 N K_{i j}+\nabla_{i} \beta_{j}+\nabla_{j} \beta_{i} \\
\partial_{t} K_{i j}=N & \left(R_{i j}-2 K_{i k} K_{j}^{k}+K K_{i j}-8 \pi G S_{i j}+4 \pi G g_{i j}(S-\rho)\right) \\
& -\nabla_{i} \nabla_{j} N+\beta^{k} \nabla_{k} K_{i j}+K_{i k} \nabla_{j} \beta^{k}+K_{k j} \nabla_{i} \beta^{k} .
\end{aligned}
$$

Here, $\nabla_{i}$ and $R$ are the covariant derivative and the scalar curvature (trace of the Ricci tensor) of $g_{i j}$, respectively, and $K=K_{i j} g^{i j}$ denotes the mean curvature. Furthermore, $G$ stands for Newton's constant, $\rho$ and $S_{i j}$ are matter density and stress tensor, respectively, and $S=S_{i j} g^{i j}$ denotes the trace of $S_{i j}$.

The constraint equations are conditions within each hypersurface alone, ensuring that the three-dimensional surface can be embedded into the four-dimensional 
spacetime:

$$
\begin{aligned}
R+K^{2}-K_{i j} K^{i j} & =16 \pi G \rho, \\
\nabla_{j}\left(K^{i j}-g^{i j} K\right) & =8 \pi G j^{i},
\end{aligned}
$$

with $j^{i}$ denoting the matter momentum density. Equation (2.4) is called the Hamiltonian constraint, and Eq. (2.5) is the momentum constraint.

Cauchy initial data for Einstein's equations consists of $\left(g_{i j}, K^{i j}\right)$ on one hypersurface satisfying the constraint equations (2.4) and (2.5). After choosing lapse and shift (which are arbitrary and merely choose a specific coordinate system), Eqs. 2.2. and (2.3) determine $\left(g_{i j}, K^{i j}\right)$ at later times. Analytically, the constraints equations are preserved under the evolution. In practice, however, during numerical evolution of Eqs. (2.2) and (2.3) or any other formulation of Einstein's equations, many problems arise.

The constraints (2.4) and (2.5) restrict four of the twelve degrees of freedom of $\left(g_{i j}, K^{i j}\right)$. As these equations are not of any standard mathematical form, it is not obvious which four degrees of freedom are restricted. Hence, finding any solutions is not trivial, and it is even harder to construct specific solutions that represent certain astrophysically relevant situations like a binary black hole.

\subsection{Preliminaries}

Both Hamiltonian and Lagrangian viewpoints use a conformal transformation on the spatial metric,

$$
g_{i j}=\psi^{4} \tilde{g}_{i j}
$$

with strictly positive conformal factor $\psi . \tilde{g}_{i j}$ is referred to as the conformal metric. From (2.6) it follows that the Christoffel symbols of the physical and conformal metrics are related by

$$
\Gamma_{j k}^{i}=\tilde{\Gamma}_{j k}^{i}+2 \psi^{-1}\left(\delta_{j}^{i} \partial_{k} \psi+\delta_{k}^{i} \partial_{j} \psi-\tilde{g}_{j k} \tilde{g}^{i l} \partial_{l} \psi\right),
$$

which in turn implies that the scalar curvatures of $g_{i j}$ and $\tilde{g}_{i j}$ are related by

$$
R=\psi^{-4} \tilde{R}-8 \psi^{-5} \tilde{\nabla}^{2} \psi
$$

Equations (2.6) - 2.8 were already known to Eisenhart [19]. Furthermore, for any symmetric tracefree tensor $\tilde{S}^{i j}$,

$$
\nabla_{j}\left(\psi^{-10} \tilde{S}^{i j}\right)=\psi^{-10} \tilde{\nabla}_{j} \tilde{S}^{i j}
$$

where $\tilde{\nabla}$ is the covariant derivative of $\tilde{g}_{i j}$. Lichnerowicz [30] used Eqs. (2.6) to (2.9) to treat the initial value problem on maximal slices, $K=0$. For non-maximal slices, the extrinsic curvature is split into trace and tracefree parts [34,

$$
K^{i j}=A^{i j}+\frac{1}{3} g^{i j} K
$$


With (2.8) and (2.10), the Hamiltonian constraint (2.4) becomes

$$
\tilde{\nabla}^{2} \psi-\frac{1}{8} \psi \tilde{R}-\frac{1}{12} \psi^{5} K^{2}+\frac{1}{8} \psi^{5} A_{i j} A^{i j}+2 \pi G \psi^{5} \rho=0,
$$

a quasi-linear Laplace equation for $\psi$. Local uniqueness proofs of equations like (2.11) often linearize around an (assumed) solution, and apply the maximum principle. However, the signs of the last two terms of (2.11) are such that the maximum principle cannot be applied and consequently, it is not immediately guaranteed that Eq. (2.11) has locally unique solutions. The term proportional to $A_{i j} A^{i j}$ will be dealt with later; for the matter terms we follow York [52] and introduce conformally scaled source terms:

$$
\begin{gathered}
j^{i}=\psi^{-10} \tilde{\jmath}^{i}, \\
\rho=\psi^{-8} \tilde{\rho} .
\end{gathered}
$$

The scaling for $j^{i}$ makes the momentum constraint below somewhat nicer; the scalings of $\rho$ and $j^{i}$ are tied together such that the dominant energy condition preserves sign:

$$
\rho^{2}-g_{i j} j^{i} j^{j}=\psi^{-16}\left(\tilde{\rho}^{2}-\tilde{g}_{i j} \tilde{\jmath}^{i} \tilde{\jmath}^{j}\right) \geq 0 .
$$

With Eq. (2.13), the matter term in (2.11) becomes $2 \pi G \psi^{-3} \tilde{\rho}$ with negative semidefinite linearization for $\tilde{\rho} \geq 0$.

The decomposition of $K_{i j}$ into trace and tracefree part, Eq. (2.10), turns the momentum constraint (2.5) into

$$
\nabla_{j} A^{i j}-\frac{2}{3} \nabla^{i} K=8 \pi G j^{i} .
$$

The conformal transformation (2.6) implies one additional conformal scaling relation. The longitudinal operator [16 5051]

$$
(\mathbb{L} V)^{i j} \equiv \nabla^{i} V^{j}+\nabla^{j} V^{i}-\frac{2}{3} g^{i j} \nabla_{k} V^{k},
$$

satisfies [50]

$$
(\mathbb{L} V)^{i j}=\psi^{-4}(\tilde{\mathbb{L}} V)^{i j} .
$$

Here $(\tilde{\mathbb{L}} V)^{i j}$ is given by the same formula (2.16) but with quantities associated with the conformal metric $\tilde{g}_{i j}$. (In fluid dynamics $(\mathbb{L} V)^{i j}$ is twice the shear of the velocity field $V^{i}$ ). In $d$ spatial dimensions, the factor $2 / 3$ in Eq. (2.16) is replaced by $2 / d$; Eq. (2.17) holds for all $d$.

\subsection{Lagrangian picture - Conformal thin sandwich formalism}

The conformal thin sandwich formalism [53] deals with the conformal metric and its time derivative; as illustrated in Figure 1 we deal with two hypersurfaces separated by an infinitesimal $\delta t$ (explaining the name "thin sandwich"), and connected by lapse $N$ and shift $\beta^{i}$. The mean curvature of each hypersurface is given by $K$ and 
The initial value problem in numerical relativity 5

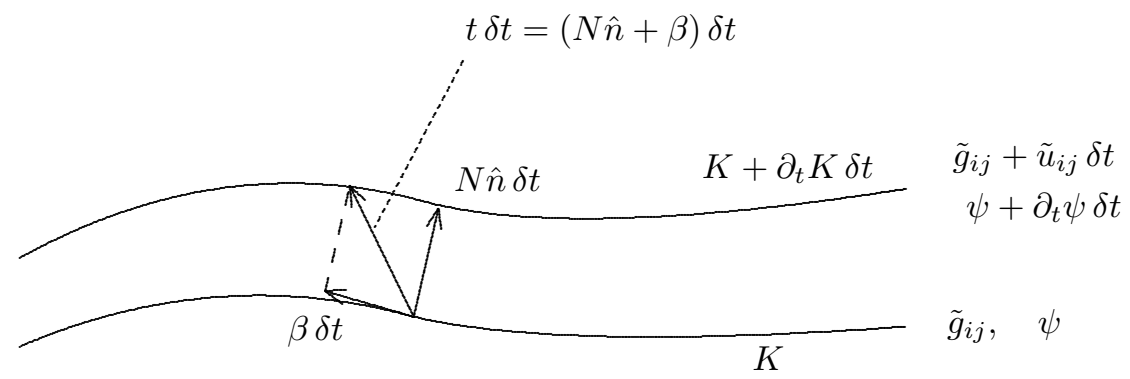

Fig. 1. Setup for conformal thin sandwich formalism.

$K+\partial_{t} K \delta t$, respectively, and the metric is decomposed into conformal factor and conformal metric. This decomposition is synchronized between the two hypersurfaces by the requirement that the conformal metrics on both hypersurfaces have the same determinant to first order in $\delta t$. The variation of the determinant of $\tilde{g}_{i j}$ is

$$
\delta \tilde{g}=\tilde{g} \tilde{g}^{i j} \delta \tilde{g}_{i j}=\tilde{g} \tilde{g}^{i j} \tilde{u}_{i j} \delta t,
$$

so that $\tilde{u}_{i j} \equiv \partial_{t} \tilde{g}_{i j}$ must be traceless.

Besides the relationships indicated in Figure 1 the conformal thin sandwich formalism rests on the nontrivial scaling behavior of the lapse function:

$$
N=\psi^{6} \tilde{N} .
$$

Indications suggesting this scaling appear in a bewildering variety of contexts (see discussion in 5340). This scaling is also crucial in the present context as well, cf. Eq. (2.24) below.

Substitution of Eq. (2.6) into the evolution equation for the metric, Eq. (2.2), and splitting into trace and trace-free parts with respect to the physical inverse metric $g^{i j}=\psi^{-4} \tilde{g}^{i j}$ results in

$$
\begin{aligned}
\partial_{t} \ln \psi & =-\frac{1}{6} N K+\frac{1}{6} \nabla_{k} \beta^{k}, \\
\psi^{4} \tilde{u}_{i j} & =-2 N A_{i j}+(\mathbb{L} \beta)_{i j} .
\end{aligned}
$$

Equation (2.21) is the tracefree piece of $\partial_{t} g_{i j}$, thus for $u_{i j} \equiv \psi^{4} \tilde{u}_{i j}$,

$$
u_{i j}=\partial_{t} g_{i j}-\frac{1}{3} g_{i j} g^{k l} \partial_{t} g_{k l} .
$$

We solve Eq. (2.21) for $A^{i j}$,

$$
A^{i j}=\frac{1}{2 N}\left((\mathbb{L} \beta)^{i j}-u^{i j}\right),
$$

and rewrite with conformal quantities [using (2.19), (2.17) and $u^{i j}=\psi^{-4} \tilde{u}^{i j}$ :

$$
A^{i j}=\psi^{-10} \frac{1}{2 \tilde{N}}\left((\tilde{\mathbb{L}} \beta)^{i j}-\tilde{u}^{i j}\right) \equiv \psi^{-10} \tilde{A}^{i j},
$$


which defines the conformal tracefree extrinsic curvature $\tilde{A}^{i j}$. Equation (2.24) shows that the formula for $A^{i j}$ is form invariant under conformal transformations; this hinges on the scaling of $N$ in Eq. (2.19). Substitution of Eq. (2.24) into the momentum constraint (2.15) and application of Eq. (2.9) yields

$$
\tilde{\nabla}_{j}\left(\frac{1}{2 \tilde{N}}(\tilde{\mathbb{L}} \beta)^{i j}\right)-\tilde{\nabla}_{j}\left(\frac{1}{2 \tilde{N}} \tilde{u}^{i j}\right)-\frac{2}{3} \psi^{6} \tilde{\nabla}^{i} K=8 \pi G \tilde{\jmath}^{i},
$$

whereas Eq. (2.24) modifies the Hamiltonian constraint (2.11) to

$$
\tilde{\nabla}^{2} \psi-\frac{1}{8} \psi \tilde{R}-\frac{1}{12} \psi^{5} K^{2}+\frac{1}{8} \psi^{-7} \tilde{A}_{i j} \tilde{A}^{i j}=-2 \pi G \psi^{-3} \tilde{\rho} .
$$

Equations 2.25) and (2.26) constitute elliptic equations for $\beta^{i}$ and $\psi$. We can therefore construct a valid initial data set as follows: Choose the free data

$$
\left(\tilde{g}_{i j}, \tilde{u}_{i j}, \quad K, \tilde{N}\right)
$$

(and matter terms if applicable), solve Eqs. (2.25) and (2.26) for $\beta^{i}$ and $\psi$, and finally, assemble $g_{i j}=\psi^{4} \tilde{g}_{i j}$ and $K^{i j}=\psi^{-10} \tilde{A}^{i j}+\frac{1}{3} g^{i j} K$.

We now comment on several issues related to the conformal thin sandwich formalism.

\subsubsection{Fixing $\tilde{N}$ via $\partial_{t} K$}

In the free data Eq. (2.27), $\tilde{g}_{i j}$ and $\tilde{u}_{i j}=\partial_{t} \tilde{g}_{i j}$ constitute a "variable \& velocity pair" $(q, \dot{q})$ in the spirit of Lagrangian mechanics, but the remaining free data does not. To improve this situation, we note that the trace of the evolution equation (2.3), results in (see, e.g., 45])

$$
\partial_{t} K-\beta^{k} \partial_{k} K=N\left(R+K^{2}+4 \pi G(S-3 \rho)\right)-\nabla^{2} N .
$$

Elimination of $R$ with the Hamiltonian constraint (2.4) and rewriting this equation with conformal quantities results in

$$
\begin{aligned}
\tilde{\nabla}^{2} \tilde{N}+14 \tilde{\nabla}^{i} \ln \psi \tilde{\nabla}_{i} \tilde{N}+\tilde{N}\left[\frac{3}{4} \tilde{R}+\frac{1}{6} \psi^{4} K^{2}-\frac{7}{4} \psi^{-8} \tilde{A}_{i j} \tilde{A}^{i j}\right. \\
\left.+42 \tilde{\nabla}_{i} \ln \psi \tilde{\nabla}^{i} \ln \psi-4 \pi G \psi^{4}(S+4 \rho)\right]=-\psi^{-2}\left(\partial_{t} K-\beta^{k} \partial_{k} K\right) .
\end{aligned}
$$

For given $\partial_{t} K$, this constitutes an elliptic equation for $\tilde{N}$. Therefore, if we take $\partial_{t} K$ as the "free" quantity instead of $\tilde{N}$, then the free data for the conformal thin sandwich formalism becomes

$$
\left(\tilde{g}_{i j}, \tilde{u}_{i j}, \quad K, \partial_{t} K\right)
$$

plus matter terms if applicable. These free data consist completely of $(q, \dot{q})$ pairs as appropriate for the Lagrangian viewpoint. These free data are also useful in practice for computations of quasi-equilibrium initial data, for which $\partial_{t} K=0$ is a natural 
and simple choice, whereas it is not obvious which conformal lapse $\tilde{N}$ should be used. We note that Eq. 2.29) can be rewritten as

$$
\begin{aligned}
\tilde{\nabla}^{2}\left(\tilde{N} \psi^{7}\right)-\left(\tilde{N} \psi^{7}\right)\left[\frac{1}{8} \tilde{R}+\frac{5}{12} \psi^{4} K^{2}+\frac{7}{8} \psi^{-8} \tilde{A}_{i j} \tilde{A}^{i j}\right. & \left.+2 \pi G \psi^{4}(\rho+2 S)\right] \\
& =-\psi^{5}\left(\partial_{t} K-\beta^{k} \partial_{k} K\right) .
\end{aligned}
$$

This equation is somewhat shorter and computationally somewhat more convenient. Using the free data (2.30), five coupled elliptic equations have to be solved, rather than four, namely (2.25), (2.26) and either (2.29) or (2.31).

\subsubsection{Invariance to conformal transformations of the free data}

Given free data (2.27) and a solution $\left(\psi, \beta^{i}\right)$ of the conformal thin sandwich equations, choose a function $\Psi>0$, and define conformally rescaled free data by

$$
\tilde{g}_{i j}^{\prime}=\Psi^{-4} \tilde{g}_{i j}, \quad \tilde{u}^{\prime i j}=\Psi^{4} \tilde{u}^{i j}, \quad K^{\prime}=K, \quad \tilde{N}^{\prime}=\Psi^{-6} \tilde{N},
$$

plus the scalings $\tilde{\rho}^{\prime}=\Psi^{8} \tilde{\rho}, \quad \tilde{\jmath}^{\prime i}=\Psi^{10} \tilde{\jmath}^{i}$ for matter terms if applicable. These free data, together with conformal factor $\psi^{\prime}=\Psi \psi$ and the shift $\beta^{\prime i}=\beta^{i}$ lead to the same physical initial data $\left(g_{i j}, K^{i j}\right)$ :

$$
\begin{aligned}
g_{i j}^{\prime} & =\psi^{\prime 4} \tilde{g}_{i j}^{\prime}=\psi^{4} \tilde{g}_{i j}=g_{i j}, \\
A^{\prime i j} & =\psi^{\prime-10} \frac{1}{2 \tilde{N}^{\prime}}\left(\left(\tilde{\mathbb{L}}^{\prime} \beta^{\prime}\right)^{i j}-\tilde{u}^{\prime i j}\right)=A^{i j} .
\end{aligned}
$$

Here, we used Eqs. (2.6) and (2.24), and $\tilde{\mathbb{L}}^{\prime}$ denotes the longitudinal operator of $\tilde{g}_{i j}^{\prime}$, which, by Eq. (2.17), satisfies $\left(\tilde{\mathbb{L}}^{\prime} \beta\right)^{i j}=\Psi^{4}(\tilde{\mathbb{L}} \beta)^{i j}$. Adding the trace of the extrinsic curvature to Eq. (2.34) is trivial.

Therefore, only the conformal equivalence class of $\tilde{g}_{i j}$ is relevant for the physical solution. This is a very desirable property; we introduced $\tilde{g}_{i j}$ as a conformal metric, so its overall scaling should not matter. Specification of $\partial_{t} K$ instead of $\tilde{N}$ as part of the free data preserves this invariance, as the $\partial_{t} K$-equation, (2.29), is derived from physical quantities in the first place.

The extrinsic curvature decomposition introduced in the next section is also invariant under conformal transformations of the free data. We note that invariance under conformal transformations of the free data is not trivial; earlier variants of the constraint decompositions did not possess it, giving rise to ambiguities in the free data which influenced numerical investigations [46].

\subsubsection{Gauge degrees of freedom}

The physical initial data $\left(g_{i j}, K^{i j}\right)$ has twelve degrees of freedom (matter just adds four additional degrees of freedom in $\tilde{\rho}$ and $\tilde{\jmath}^{i}$ which determine the four physical matter variables $\rho$ and $j^{i}$ ). It is constrained by four constraint equations, so there should be eight degrees of freedom in the freely specifiable data. However, even 
taking into account that only the conformal equivalence class of $\tilde{g}_{i j}$ is relevant and that $\tilde{u}_{i j}$ is traceless, the free data (2.27) or (2.30) consists of twelve quantities, not eight. To clarify this issue, consider the substitutions

$$
\begin{aligned}
\tilde{u}^{i j} & \rightarrow \tilde{u}^{i j}+(\tilde{\mathbb{L}} W)^{i j}, \\
\beta^{i} & \rightarrow \beta^{i}+W^{i} .
\end{aligned}
$$

The vector $W^{i}$ disappears from Eqs. (2.23)-(2.26), therefore the substitution (2.35) will not change the physical initial data set $\left(g_{i j}, K^{i j}\right)$; it merely tilts the time-axis and changes the coordinate labels on the second hypersurface. Thus, $\tilde{u}_{i j}$ contains three gauge degrees of freedom associated with the shift. ( $W^{i}$ enters into Eq. (2.29) as an advection term, though, because $\partial_{t} K$ is the derivative along the time-vector).

The fourth "missing" degree of freedom is hidden in the lapse function $\tilde{N}$ : One can construct every possible initial data set $\left(g_{i j}, K^{i j}\right)$ with any (non-pathologic) choice of $\tilde{N}$. This can be seen by going backward from the physical initial data $\left(g_{i j}, K^{i j}\right)$ (satisfying the constraints) to the free data. Given $\left(g_{i j}, K^{i j}\right)$ and any $\tilde{N}$ and $\beta^{i}$, set the free data (2.27) by

$$
\tilde{g}_{i j}=g_{i j}, \quad \tilde{u}^{i j}=(\mathbb{L} \beta)^{i j}-2 \tilde{N} A^{i j}, \quad K=K^{i j} g_{i j}
$$

as well as the given $\tilde{N}$. With these free data, $\psi \equiv 1$ and the given $\beta^{i}$ will reconstruct the physical spacetime $\left(g_{i j}, K^{i j}\right)$ as can be seen from Eqs. (2.6) and (2.24). Therefore, $\psi \equiv 1$ and the given $\beta^{i}$ will solve the conformal thin sandwich equations (2.25) and (2.26).

The fact that we were free to choose $\beta^{i}$ reflects again the gauge-symmetry illustrated in Eq. (2.35), but in addition, we showed that the choice of $\tilde{N}$ does not restrict the set of "reachable" initial data sets.

Note that the physical initial data contain further gauge freedom: Covariance under spatial transformations implies that $g_{i j}$ (and $\tilde{g}_{i j}$ ) contain three gauge degrees of freedom associated with the choice of coordinates. Furthermore, $K$ can be interpreted as time [49, fixing the temporal gauge. Thus, an initial data set has only four physical degrees of freedom - in perturbed flat space they are simply the two polarizations of gravitational waves.

\subsubsection{Implications for an evolution of the initial data}

During the solution of the conformal thin sandwich equations, one finds a shift $\beta^{i}$ and a lapse $N$. If this gauge is used in a subsequent evolution of the initial data $\left(g_{i j}, K^{i j}\right)$ then Eq. (2.22) implies that, initially,

$$
\partial_{t} g_{i j}-\frac{1}{3} g_{i j} g^{k l} \partial_{t} g_{k l}=\psi^{4} \tilde{u}_{i j} .
$$

The freely specifiable piece $\tilde{u}_{i j}$ thus directly controls the tracefree part of the timederivative of the metric. If we specified $\partial_{t} K$ as part of the free data, then, of course, this will be the initial time-derivative of the mean curvature. Finally, from (2.20), 
we find

$$
\begin{aligned}
\partial_{t} \ln \psi & =\frac{1}{6}\left(\nabla_{k} \beta^{k}-N K\right) \\
& =\frac{1}{6} \tilde{\nabla}_{k} \beta^{k}+\beta^{k} \partial_{k} \ln \psi-\frac{1}{6} \psi^{6} \tilde{N} K .
\end{aligned}
$$

Since we have been very successful so far with specification of time-derivatives $\left(\partial_{t} \tilde{g}_{i j}\right.$ and $\left.\partial_{t} K\right)$, one might be tempted to turn (2.38) around and use it as the definition of $K$ in terms of $\partial_{t} \ln \psi$. This idea certainly comes to mind when looking for quasi-equilibrium solutions, for which time-derivatives of as many quantities as possible should vanish. Pursuing this idea, we find from Eq. (2.38)

$$
K=\frac{1}{\psi^{6} \tilde{N}}\left(\tilde{\nabla}_{k} \beta^{k}-6\left(\partial_{t}-\beta^{k} \partial_{k}\right) \ln \psi\right) .
$$

Substituting Eq. (2.39) into the momentum constraint Eq. (2.25), however, makes the principal part of Eq. (2.25) proportional to

$$
\partial_{j} \partial^{j} \beta^{i}-\partial^{i} \partial_{k} \beta^{k}
$$

which is non-invertible. Therefore the attempt to fix $K$ via (2.39) will fail. Indeed, during the construction quasi-equilibrium initial data sets of spherically symmetric spacetimes 15, it was found that the conformal thin sandwich formalism with $\tilde{u}_{i j}=0$ and $\partial_{t} K=0$ (and appropriate boundary conditions) is so successful in picking out the time-like Killing vector that several different choices of $K$ lead to solutions satisfying $\partial_{t} \ln \psi=0$.

We conclude that, in contrast to the trace-free part (2.37), one can not easily control $\partial_{t} \ln \psi$ by choices of the free data. One can only evaluate (2.38) after solving the conformal thin sandwich equations.

\subsection{Hamiltonian picture - Extrinsic curvature decomposition}

The second method to construct solutions of the constraint equations if based on a decomposition of the extrinsic curvature. Early variants of this approach 3452 have been widely used for almost thirty years, but the final version was developed only very recently 40 . We will make use of the equations and results from section 2.1. in particular, we use a conformal metric, $g_{i j}=\psi^{4} \tilde{g}_{i j}$, and split the extrinsic curvature into trace and trace-free parts, $K^{i j}=A^{i j}+1 / 3 g^{i j} K$, cf. Eqs. (2.6) and (2.10).

We start with a weighted transverse traceless decomposition of $A^{i j}$,

$$
A^{i j}=A_{T T}^{i j}+\frac{1}{\sigma}(\mathbb{L} V)^{i j} .
$$

Here, $A_{T T}^{i j}$ is transverse, $\nabla_{j} A_{T T}^{i j}=0$, and traceless, $g_{i j} A_{T T}^{i j}=0$, and $\sigma$ is a strictly positive and bounded function. Appearance of the weight function $\sigma$ is a key point in the extrinsic curvature formulation; its inclusion is the major difference of [40] over the older variants. 
Given a symmetric tracefree tensor like $A^{i j}$, the decomposition (2.41) is obtained by taking the divergence of Eq. (2.41),

$$
\nabla_{j} A^{i j}=\nabla_{j}\left[\sigma^{-1}(\mathbb{L} V)^{i j}\right] .
$$

The right hand side, $\nabla_{j}\left[\sigma^{-1}(\mathbb{L} .)^{i j}\right]$, is a well-behaved elliptic operator in divergence form, so no problem should arise when solving (2.42) for $V^{i}$. Substitution of the solution $V^{i}$ back into (2.41) yields $A_{T T}^{i j}$. In the presence of boundaries, Eq. (2.42) requires boundary conditions which will influence the solution $V^{i}$ and the decomposition (2.41). For closed manifolds, existence and uniqueness of the decomposition (2.41) for the case $\sigma \equiv 1$ was shown in [51].

We now conformally scale the quantities on the right hand side of (2.41) with the goal of rewriting the momentum constraint in conformal space. First, we set

$$
A_{T T}^{i j} \equiv \psi^{-10} \tilde{A}_{T T}^{i j} .
$$

Equation (2.9) ensures that $\tilde{A}_{T T}^{i j}$ is transverse with respect to $\tilde{g}_{i j}$ if and only if $A_{T T}^{i j}$ is transverse with respect to the physical metric $g_{i j}$. Because of Eq. (2.17), and because $\mathbb{L}$ is the conformal Killing operator, the vector $V^{i}$ is not rescaled. The conformal scaling of the weight function is given by

$$
\sigma=\psi^{6} \tilde{\sigma} .
$$

The most immediate reason for this scaling is to allow Eq. 2.45) below; several more reasons will be mentioned in the sequel.

Using the scaling relations (2.17), (2.43) and (2.44), we can rewrite Eq. (2.41) as

$$
A^{i j}=\psi^{-10}\left(\tilde{A}_{T T}^{i j}+\frac{1}{\tilde{\sigma}}(\tilde{\mathbb{L}} V)^{i j}\right)=\psi^{-10} \tilde{A}^{i j},
$$

where

$$
\tilde{A}^{i j} \equiv \tilde{A}_{T T}^{i j}+\tilde{\sigma}^{-1}(\tilde{\mathbb{L}} V)^{i j}
$$

is a weighted transverse traceless decomposition in the conformal space. The scaling $A^{i j}=\psi^{-10} \tilde{A}^{i j}$ was not postulated (as it had to be in the old variants), but follows from the other scalings. By virtue of the scaling of the weight function $\sigma$, Eq. (2.44), the weighted transverse traceless decomposition thus commutes with the conformal transformation. This commutation of conformal transformation and weighted transverse traceless decomposition is precisely the new feature of the weighted decomposition. Without the weight-function, conformal transformations and transversetraceless decomposition do not commute, leading to the two inequivalent old variants, depending on which operation is performed first.

Equations (2.9) and (2.45) allow us to rewrite the momentum constraint (2.15) as

$$
\tilde{\nabla}_{j}\left(\frac{1}{\tilde{\sigma}}(\tilde{\mathbb{L}} V)^{i j}\right)-\frac{2}{3} \psi^{6} \tilde{\nabla}^{i} K=8 \pi G \tilde{\jmath}^{i},
$$


an elliptic equation for $V^{i}$. The Hamiltonian constraint Eq. (2.11) reads

$$
\tilde{\nabla}^{2} \psi-\frac{1}{8} \tilde{R} \psi-\frac{1}{12} \psi^{5} K^{2}+\frac{1}{8} \psi^{-7} \tilde{A}_{i j} \tilde{A}^{i j}=-2 \pi G \psi^{-3} \tilde{\rho},
$$

with $\tilde{A}^{i j}$ given by Eq. (2.46). Equation (2.48) is identical to Eq. (2.26) since in both formulations $A^{i j}=\psi^{-10} \tilde{A}^{i j}$, however, the definitions of $\tilde{A}^{i j}$ differ.

Starting from the physical initial data $\left(g_{i j}, K^{i j}\right)$, we have now rewritten the constraints (2.4) and (2.5) as elliptic equations (2.47) and (2.48). In order to construct a valid initial data set $\left(g_{i j}, K^{i j}\right)$, one first chooses the free data

$$
\left(\tilde{g}_{i j}, K, \tilde{A}_{T T}^{i j}, \tilde{\sigma}\right)
$$

and matter terms if applicable, then solves Eqs. (2.47) and (2.48) for $V^{i}$ and $\psi$, and finally assembles the physical solution by Eqs. (2.6), (2.10), and (2.45).

\subsubsection{Remarks on the extrinsic curvature decomposition}

Similar to section 2.2.2 one can show that the physical initial data $\left(g_{i j}, K^{i j}\right)$ is invariant to a conformal transformation of the free data. For $\Psi>0$, the relevant transformations are [cf. Eq. [2.32)]:

$$
\tilde{g}_{i j}^{\prime}=\Psi^{-4} \tilde{g}_{i j}, \quad \tilde{A}_{T T}^{\prime i j}=\Psi^{10} A_{T T}^{i j}, \quad K^{\prime}=K, \quad \tilde{\sigma}^{\prime}=\Psi^{-6} \tilde{\sigma}
$$

plus the scalings $\tilde{\rho}^{\prime}=\Psi^{8} \tilde{\rho}, \quad \tilde{\jmath}^{\prime i}=\Psi^{10} \tilde{\jmath}^{i}$ for matter terms if applicable. The calculation is straightforward, the key-point being that the scaling of the weightfunction (2.44) synchronizes the conformal scaling of the transverse-traceless and longitudinal parts of the weighted transverse traceless decomposition.

Because of the invariance to conformal scalings of the free data, $\tilde{g}_{i j}$ supplies only five degrees of freedom, so that the free data Eq. 2.49) contains nine degrees of freedom. The weight $\sigma$ (or $\tilde{\sigma}$ ) merely parametrizes the transverse traceless decomposition (2.41). For any choice of $\sigma$, the decomposition (2.41) can be performed, therefore with any choice of $\tilde{\sigma}$, all initial data sets can be generated for appropriate choices of the free data.

To construct a transverse traceless tensor $\tilde{A}_{T T}^{i j}$ compatible with the metric $\tilde{g}_{i j}$, one decomposes a general symmetric tracefree tensor $\tilde{M}^{i j}$. Write

$$
\tilde{M}^{i j}=\tilde{A}_{T T}^{i j}+\frac{1}{\tilde{\sigma}}(\tilde{\mathbb{L}} W)^{i j} .
$$

The divergence of this equation,

$$
\tilde{\nabla}_{j} \tilde{M}^{i j}=\tilde{\nabla}_{j}\left[\tilde{\sigma}^{-1}(\tilde{\mathbb{L}} W)^{i j}\right],
$$

represents an elliptic equation for $W^{i}$. Solving this equation, and substituting $W^{i}$ back into (2.51) yields

$$
\tilde{A}_{T T}^{i j}=\tilde{M}^{i j}-\frac{1}{\tilde{\sigma}}(\tilde{\mathbb{L}} W)^{i j}
$$


The formula for $\tilde{A}^{i j}$, Eq. (2.46), now reads

$$
\tilde{A}^{i j}=\tilde{M}^{i j}+\frac{1}{\tilde{\sigma}}[\tilde{\mathbb{L}}(V-W)]^{i j},
$$

which depends only on the difference $V^{i}-W^{i}$. On the other hand, subtraction of (2.52) from the momentum constraint (2.47) yields

$$
\tilde{\nabla}_{j}\left(\frac{1}{\tilde{\sigma}}[\tilde{\mathbb{L}}(V-W)]^{i j}\right)+\tilde{\nabla}_{j} \tilde{M}^{i j}-\frac{2}{3} \psi^{6} \tilde{\nabla}^{i} K=8 \pi G \tilde{\jmath}^{i},
$$

which is an equation for the difference $V^{i}-W^{i}$. Thus one can combine the construction of $\tilde{A}_{T T}^{i j}$ from $\tilde{M}^{i j}$ with the solution of the momentum constraint, as observed by Cantor [48. Instead of solving (2.52) for $W^{i}$ and then (2.47) for $V^{i}$, one can directly solve (2.55) for $V^{i}-W^{i}$.

In the presence of boundaries, solutions of elliptic equations like (2.55) or (2.52) will depend on boundary conditions. When constructing black hole initial data, inner boundaries are often present, and it is far from clear what boundary conditions to apply there. In Ref. 36, for example, the situation is encountered that boundary conditions for the combined solution for $V^{i}-W^{i}$ are known, but not for the individual solutions for $W^{i}$ and $V^{i}$.

\subsubsection{Identification of $\sigma$ with the lapse $N$}

The extrinsic curvature formulation of the initial value problem as presented so far is perfectly adequate for the mathematical task of rewriting the constraints as welldefined equations. However, it is very natural to further identify the weight-function $\sigma$ with the lapse function $N$,

$$
\sigma=2 N, \quad \tilde{\sigma}=2 \tilde{N} .
$$

One reason for this identification is that $\sigma$ and $N$ have the same conformal scaling behavior, cf. Eqs. (2.19) and (2.44). A second reason is that with this identification, the conformal thin sandwich equations become equivalent to the extrinsic curvature formulation. To see this, note that by virtue of (2.56), Eqs. (2.54) and (2.55) become

$$
\tilde{A}^{i j}=\tilde{M}^{i j}+\frac{1}{2 \tilde{N}}[\tilde{\mathbb{L}}(V-W)]^{i j},
$$

and

$$
\tilde{\nabla}_{j}\left(\frac{1}{2 \tilde{N}}[\tilde{\mathbb{L}}(V-W)]^{i j}\right)+\tilde{\nabla}_{j} \tilde{M}^{i j}-\frac{2}{3} \psi^{6} \tilde{\nabla}^{i} K=8 \pi G \tilde{\jmath}^{i} .
$$

With the identifications

$$
\tilde{M}^{i j} \leftrightarrow-\frac{1}{2 \tilde{N}} \tilde{u}^{i j}, \quad V^{i}-W^{i} \leftrightarrow \beta^{i},
$$

Eqs. (2.57) and (2.58) are identical to Eqs. (2.24) and (2.25) of the conformal thin sandwich formalism. The Lagrangian picture agrees completely with the Hamiltonian picture. A third reason for (2.56) is given next. 


\subsubsection{Stationary spacetimes have $A_{T T}^{i j}=0$}

Consider a stationary solution of Einstein's equations with timelike Killing vector $l$. Given a spacelike hypersurface $\Sigma$, there is a preferred gauge so that the time-vector of an evolution coincides with $l$, namely $N=-n \cdot l, \beta=\perp l$, where $n$ is the unit normal to $\Sigma$, and $\perp$ is the projection operator into $\Sigma$. With this choice of lapse and shift, $g_{i j}$ and $K^{i j}$ will be time-independent. Using $\partial_{t} g_{i j}=0$ in Eq. (2.2) and taking the tracefree part yields

$$
A^{i j}=\frac{1}{2 N}(\mathbb{L} \beta)^{i j},
$$

a weighted transverse traceless decomposition with $A_{T T}^{i j} \equiv 0$. Thus, with the appropriate weight factor $\sigma=2 N$, the extrinsic curvature has no transverse traceless piece for any spacelike slice in any spacetime with timelike Killing vector (A similar argument is applicable in the ergosphere of a Kerr black hole; however, one must be more careful with the choice of $\Sigma$ relative to $l$ ).

This is an important result. One generally identifies the transverse traceless piece of the extrinsic curvature with radiative degrees of freedom. Stationary spacetimes do not radiate, and therefore $A_{T T}^{i j}$ should indeed vanish. In contrast, a transversetraceless decomposition of $A^{i j}$ without the weight-factor will in general lead to a nonzero transverse traceless piece so that such a decomposition is incompatible with the identification of $A_{T T}^{i j}$ with "gravitational radiation."

\section{Binary black hole initial data}

Up to about five years ago, many assumptions were necessary to simplify the initial value problem sufficiently to make it tractable for the computational methods of that time. The major assumptions were (see 12] for a review)

(1) Maximal slicing, $K=0$.

(2) Conformal flatness $\tilde{g}_{i j}=f_{i j}$, where $f_{i j}$ represents the Euclidean metric.

(3) Use of the analytical Bowen-York [6] extrinsic curvature to solve the momentum constraint.

Under these assumptions, only a single quasi-linear (flat-space) Laplace-equation must to be solved numerically for the conformal factor. This was done, e.g., with inversion symmetry boundary conditions [1114] or with the puncture method [8]. Since then, the conformal method (as presented in Sec. 2) was completed, and several numerical codes were developed that are capable of solving the coupled constraint equations 312325384647. Three different numerical techniques have been used to discretize the elliptic problems, finite differences, spectral methods and finite elements.

Finite differences 314647] are familiar to almost all researchers, and are fairly straightforward to implement. However, the presence of different length scales

$$
M_{A, B} \ll d \ll R,
$$


where $M_{A, B}$ represents the masses of the two black holes (labeled $A$ and $B$ ), $d$ their separation and $R$ the distance to the outer boundary of the computational grid, restricts finite difference codes on uniform grids to a very coarse resolutions with errors of $10^{-2}$ to $10^{-3}$. Imposing boundary conditions on spherical inner boundaries is also difficult in finite difference codes. Adaptive mesh-refinement may drastically improve the ability of finite-difference codes to handle the different length scales in Eq. (3.1). In Refs. 17419] the Hamiltonian constraint alone is solved, and work is in progress to extend adaptive mesh refinement to the coupled initial value equations 42 .

Spectral methods 2338 (see 107 for general introductions) approximate the solution with a truncated expansion in some basis functions, typically Chebyshevpolynomials or spherical harmonics. The solutions to the constraint equations are smooth, so that the accuracy improves exponential with the number of basis functions and much higher accuracies are achieved $\left(\sim 10^{-9}\right.$ for binary black hole initial data in 38). Spectral methods are also more efficient, therefore permitting much larger parameter studies, allow more easily to impose boundary conditions on spherical boundaries (for expansions in spherical harmonics), and, in combination with domain-decomposition techniques, easily resolve the different length scales in Eq. (3.1). The most accurate (for binary black holes) and versatile code seems to be the one developed by the author [38, which has been used to solve essentially all versions of the initial value problem 36351537 .

Finite elements [2514] cover the computational domain with very many small computational cells (typically tedrahedra), and expand the solution to low polynomial order (often just linear) in each cell. The method presented in Refs. 254] is capable of solving the coupled constraint equations, and work toward using it for physically meaningful initial data is under way.

With these new codes and with the final conformal method it is possible to move beyond the assumptions mentioned above. In particular, three separate issues have been pursued: Exploration of conformally non-flat three-geometries, which is motivated by the facts that the Kerr spacetime does not admit conformally flat slices 332129], and that a binary compact object is not conformally flat at second post-Newtonian order 43. Replacement of the Bowen-York extrinsic curvature, which does not exactly reproduce a stationary spinning or boosted black hole, and which may be responsible for unexpected behavior of sequences of circular orbits for spinning equal-mass black holes [39] or irrotational black holes in the test-mass limit 35. And finally, physically motivated boundary conditions at inner excision surfaces surrounding the singularities of the black holes.

The goal of these new investigations is the construction of astrophysically realistic binary black hole data, i.e. configurations as they occur during the inspiral of two black holes in nature. Ideally, of course, such an initial data set should contain the outgoing gravitational wave signal of the preceding inspiral. However, at the current stage of sophistication, this is not taken into account, and the intermediate 
goal is to construct initial data with as little "spurious," "unphysical" gravitational energy content as possible. The quotes used around the terms "spurious" and "unphysical" indicate part of the challenge: It is not even clear what these terms mean precisely on a single spacelike surface.

Some of the research discussed in this section predates the conformal thin sandwich formalism, or the final extrinsic curvature decomposition as discussed in Sec.2] These earlier papers typically use special cases of these more general frameworks, and we will discuss them from that perspective.

\subsection{Numerical solution of the extrinsic curvature decomposition}

As mentioned above, the Kerr spacetime does not admit conformally flat slices, so that any initial data built on conformal flatness will not be able to reproduce Kerr exactly. One approach to address this issue — proposed by Matzner et al. 32 superposes exact quantities for single (spinning or boosted) black holes to define the free data for the extrinsic curvature decomposition. Because the method is built on analytical single black hole solutions, it trivially works for single black holes. Specifically, Matzner et al. considered the Kerr-Schild form of the Kerr spacetime,

$$
{ }^{(4)} \mathbf{g}_{\mu \nu}=\eta_{\mu \nu}+2 H l_{\mu} l_{\nu},
$$

where $\eta_{\mu \nu}$ represents the Minkowski metric, $H$ is a scalar function on spacetime, which decays as $1 / r$ at large radii, and $l_{\mu}$ is null with respect to both the full metric and the Minkowski metric (the concrete expressions can be found, e.g., in [32]). The Kerr-Schild form is preserved under a Lorentz-transformation, and the metric for a boosted black hole can be obtained by a suitable Lorentz-transformation on $l_{\mu}$. In order to construct initial data for a spacetime containing two black holes at coordinate locations $\vec{c}_{A, B}$ with masses $M_{A, B}$, velocities $\vec{v}_{A, B}$ and spins $M_{A} \vec{a}_{A}$ and $M_{B} \vec{a}_{B}$, one constructs the Kerr-Schild form Eq. (3.2) for each of the two black holes separately. The free data for the extrinsic curvature decomposition is then taken as the superposition

$$
\begin{aligned}
\tilde{g}_{i j} & =\delta_{i j}+2 H^{A} l_{i}^{A} l_{j}^{A}+2 H^{B} l_{i}^{B} l_{j}^{B}, \\
K & =K_{A}+K_{B} \\
\tilde{M}^{i j} & =\left(K_{A k}^{(i}+K_{B k}^{(i}-\frac{1}{3} \delta_{k}^{(i}\left(K_{A}+K_{B}\right)\right) \tilde{g}^{j) k} .
\end{aligned}
$$

Equation (3.3) is a natural generalization to two black holes of Eq. (3.2). Equation (3.5) is somewhat complicated because $\tilde{M}^{i j}$ must be tracefree with respect to the superposed metric $\tilde{g}_{i j}$, cf. Eq. (2.51). In Ref. 31 the elliptic equations of the extrinsic curvature decomposition were solved for the case $\tilde{\sigma} \equiv 1$ (i.e. one of the old variants of the extrinsic curvature decomposition).

In the limit of large separation of the black holes, one obtains two widely separated Kerr-Schild metrics, each of which, by construction will represent a (possibly) 
boosted and spinning black hole exactly, so that the superposed Kerr-Schild approach certainly is advantageous for widely separated binaries. However, the most interesting initial data is for a binary with separation close to the innermost stable circular orbit, with black hole separations of only a few Schwarzschild radii. In that case, close to the horizon of, say, hole $A$, the correction $2 H^{B} l_{i}^{B} l_{j}^{B}$ due to hole $B$ will not be small; for example, at the intersection of the line connecting the centers $\vec{c}_{A}$ and $\vec{c}_{B}$ with the horizon of hole $A$, one finds for separation $10 M$ and for non-spinning black holes,

$$
\frac{H^{B}}{H^{A}}=\frac{1}{4}
$$

Given the nonlinearities in Einsteins equations, it is not clear how the final initial data set will be influenced by this large perturbation. Since it is expected that only a few percent of the energy will be emitted in gravitational waves during the inspiral and merger of a binary black hole, it is necessary to control the energy-content of the initial data set to at least one percent of the energy (and preferably much better). In view of the ratio (3.6), it seems unlikely that this is the case. Indeed, the author 36 has examined the proposal of superposed Kerr-Schild quantities extensively with a spectral code for the construction of two black holes at rest. Using superposed Kerr-Schild quantities within the conformal thin sandwich formalism and within the extrinsic curvature formalism resulted in variations of the ADM-energy by up to several per cent. It was also found that the resulting initial data sets depend sensitively on the choice of extrinsic curvature: Removal of the longitudinal part of $\tilde{M}^{i j}$ by a procedure analogous to Eqs. (2.51) (2.54) before solving the constraint equations changes the ADM-energy by several percent.

We also note that superposed Kerr-Schild metrics singles out arbitrarily a specific slicing: Any slicing of the Kerr spacetime will give rise to three-metric and extrinsic curvatures which can be superposed similarly to Eqs. 3.3 -3.5) Furthermore, the superposition depends on the spatial coordinate system used to represent the single black hole quantities. For non-spinning, unboosted black holes, the spatial part of the Kerr-Schild metric is spherically symmetric, and can be made conformally flat by a radial coordinate transformation [13. After this spatial coordinate transformation, the equivalent of Eq. (3.3) is superposition of two conformally flat metrics, which implies that $\tilde{g}_{i j}$ should be chosen to be conformally flat 3515]. While superposing single black hole quantities is certainly an interesting route, more exhaustive investigations into their properties are necessary.

Conformal flatness is also questionable because a binary compact object is not conformally flat at second post-Newtonian order [43. Tichy et al. [46] address this issue by using post-Newtonian results to set the free data for the extrinsic curvature decomposition: The post-Newtonian expansion of the spatial metric can be written as 27.

$$
g_{i j}^{\mathrm{PN}}=\psi_{\mathrm{PN}}^{4} \delta_{i j}+h_{i j}^{T T}
$$


where $\psi_{\mathrm{PN}}$ and $h_{i j}^{T T}$ are given as expansions in the velocity $v / c$. The spatial metric Eq. (3.7) and the corresponding extrinsic curvature are known for general motion of the masses, i.e. in particular for a binary in circular orbits. The extrinsic curvature can be similarly expressed as a series in $v / c$, the leading order term being the Bowen-York momentum. One now bases the conformal metric on Eq. (3.7), and similarly for the extrinsic curvature. This approach is the only one so far, which, in principle, can account for genuine binary contributions to the free data.

Tichy et al. [46] encountered two important issues during implementation of this approach. The first problem is that close to the point-masses, the post-Newtonian expansion breaks down, that is, different terms in the expansion grow with different inverse powers of distance to the point mass. Terms of higher order in $v / c$ diverge faster than the lower order terms, so that the choice which terms one retains (e.g., consistent in the order $v / c$ ) influences the obtained initial data sets strongly. This may reflect a fundamental problem of using post-Newtonian expansions: They are least accurate close to the point-masses. The second problem encountered by Ref. [46] is related to the fact that $\psi_{\mathrm{PN}}$ has a singularity at the point-masses. In order to obtain a finite conformal metric, the authors decided to conformally scale $g_{i j}^{\mathrm{PN}}$,

$$
\tilde{g}_{i j} \equiv \Omega^{-4} g_{i j}^{\mathrm{PN}},
$$

where the leading order behavior of $\Omega$ close to the singularities is identical to that of $\psi_{\mathrm{PN}}$ However, the concrete choice for $\Omega$ influences the resulting initial data sets in a significant way; for example, the simplest choice $\Omega=\psi_{\mathrm{PN}}$ leads to binary black hole initial data sets with increasing energy as the separation between the black holes is reduced. Tichy et al. do not employ the extrinsic curvature decomposition presented in Sec. 2.3 but rather the old version without the weight-function $\sigma$ (their work predates discovery of the weight-function in Ref. 40]). This old decomposition is not invariant to conformal transformations of the free data, while the new one is [cf. Eq. [2.50]], so that ambiguities related to the choice of $\Omega$ arise only in the old decomposition. It would be interesting to repeat Tichy's work with the new decomposition.

\subsection{Numerical solutions of the conformal thin sandwich equations}

The conformal thin sandwich formalism has two advantages over the extrinsic curvature decomposition. First, it replaces the free data corresponding to the tracefree extrinsic curvature $\tilde{M}^{i j}$ and the weight function $\tilde{\sigma}$ by freely specifiable timederivatives, $\partial_{t} \tilde{g}_{i j}=\tilde{u}_{i j}$ and $\partial_{t} K$. Time-derivatives allow for easier physical interpretation of the initial data set under construction, and as we will see below, in the most interesting case, there is a natural choice for these time-derivatives, thus avoiding ambiguities related to the choice of, e.g., $\tilde{M}^{i j}$ in Eq. (3.5). As second advantage, solution of the conformal thin sandwich equations results directly in a preferred gauge choice $\left(N, \beta^{i}\right)$, which can be used in evolutions of the initial data 
set (at least during the initial stages of the evolution).

The orbits of binary compact objects of similar masses are expected to circularize before late inspiral, so that shortly before merger, the compact objects will move in circular orbits about each other. Therefore, initial data with two black holes in such a quasi-circular orbit is of particular interest. In the co-rotating coordinate system such a configuration will appear time-independent (up to corrections due to radiation reaction which are neglected so far), and so the free data corresponding to time-derivatives is simply set to zero. This motivates use of the conformal thin sandwich formalism with the free data Eq. (2.30), fully half of which consists of time-derivatives:

$$
\tilde{u}_{i j}=\partial_{t} K=0,
$$

The notion of time-independence in the corotating frame is basically equivalent to the assumption of an approximate helical Killing vector [22, which close to infinity takes the form $l_{\mathrm{HKV}}=\partial / \partial t_{0}+\Omega_{0} \partial / \partial \phi_{0}$, where $\partial / \partial t_{0}$ and $\partial / \partial \phi_{0}$ are, respectively, the asymptotic timelike and rotational Killing vectors. This follows simply from the fact that, in the corotating frame, the time-vector $t^{\mu}$ of the evolution coincides with the approximate helical Killing vector. Assuming asymptotic flatness, and requiring that the time-vector of a subsequent evolution coincides with $l_{\mathrm{HKV}}$ close to infinity leads in boundary conditions at the outer boundary,

$$
\begin{aligned}
\psi & \rightarrow 1, & r & \rightarrow \infty, \\
\beta^{i} & \rightarrow \Omega_{0}\left(\frac{\partial}{\partial \phi_{0}}\right)^{i}, & r & \rightarrow \infty, \\
N & \rightarrow 1, & r & \rightarrow \infty .
\end{aligned}
$$

It now remains to choose $\tilde{g}_{i j}$ and $K$ as well as inner boundary conditions at excision spheres around the singularities of the black holes ${ }^{\mathrm{a}}$.

The first proposal for such boundary conditions was made by Gourgoulhon et al. $22{ }^{\mathrm{b}}$, and numerical solutions were obtained with a spectral code in a companion paper 23]. Refs. 2223] assumed conformal flatness and maximal slicing to simplify the problem. Part of the necessary boundary conditions at inner boundaries were derived from the demand that the obtained initial data be inversion symmetric across the throats of the horizons, resulting in a Robin boundary condition on the conformal factor,

$$
\tilde{s}^{i} \tilde{\nabla}_{i} \psi+\frac{\psi}{2 r}=0, \quad \text { on } \mathcal{S}
$$

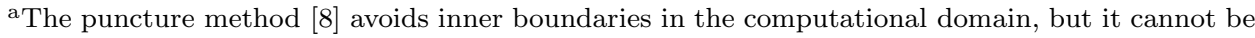
generalized to the construction of quasi-equilibrium initial data within the conformal thin sandwich formalism [24].

${ }^{\mathrm{b}}$ Gourgoulhon et al. scale the tracefree extrinsic curvature differently from Eq. 2.24), so that the elliptic equations differ in lower order terms and some of the statements made in Sec. 2.2. do not apply. 
where $r$ denotes the coordinate radius of each excised sphere, and $\tilde{s}^{i}$ represents the outward pointing (i.e. toward infinity) normal to $\mathcal{S}$, tangent to the hypersurface and normalized such that $\tilde{g}_{i j} \tilde{s}^{i} \tilde{s}^{j}=1$. Inversion symmetry also implies that the lapse function must vanish on the throat,

$$
N=0, \quad \text { on } \mathcal{S},
$$

which constitutes a boundary condition on the fifth initial value equation, Eq. (2.31). Boundary conditions on the shift, finally, were obtained by requiring that the timevector $t^{\mu}=N n^{\mu}+\beta^{\mu}$ be tangent to the generators of the horizon. In particular, $t^{\mu}$ must be null, $t^{\mu} t^{\nu(4)} \mathbf{g}_{\mu \nu}=0$, so that from Eq. (3.14) it follows

$$
\beta^{i}=0, \quad \text { on } \mathcal{S} .
$$

Since the lapse-function vanishes on $\mathcal{S}$, inspection of Eqs. (2.23) and (3.9) reveals that the extrinsic curvature will be finite on $\mathcal{S}$ only if $(\mathbb{L} \beta)^{i j}$ vanishes. However, this is not the case, so that Refs. 2223] must resort to a regularization procedure which causes the resulting initial data to violate the constraints [13] on some small level. The authors continue and construct a sequence of quasi-circular orbits for corotating black holes, and find that the location of the innermost stable circular orbit (ISCO) is close to post-Newtonian predictions.

A more general and more sophisticated approach to inner boundary conditions is due to Cook \& Pfeiffer 133515. The idea is to use the physical concept of black holes in quasi-equilibrium to derive as many boundary conditions as possible. As we will see, this approach can be used in combination with any choice for $\tilde{g}_{i j}$ and $K$. Two topological spherical regions with boundary $\mathcal{S}$ are excised from the computational domain. Denote the outward-pointing null-geodesics tangent to $\mathcal{S}$ by $k^{\mu}$, and their expansion by $\theta$. Then the following demands are made:

(1) The surfaces $\mathcal{S}$ must be apparent horizons ${ }^{\mathrm{c}}$, i.e. $\left.\theta\right|_{\mathcal{S}}=0$.

(2) The shear of $k^{\mu}$ must vanish on $\mathcal{S}$.

(3) When the initial data are evolved with the lapse and shift obtained during the solution of the conformal thin sandwich equations, then the coordinate surfaces of the apparent horizons are to remain stationary initially.

The first of these demands simply localizes the apparent horizon in coordinate space, so that it is known where to apply the other conditions. The second requirement is based on quasi-equilibrium; it implies, using Raychaudhuri's equation for nullcongruences, that

$$
\left.\mathcal{L}_{k} \theta\right|_{\mathcal{S}}=0 .
$$

That is, initially, the apparent horizon will evolve along $k^{\mu}$ and because the expansion of $k^{\mu}$ vanishes, the apparent horizon area will remain constant initially. The third demand is a gauge choice ensuring that the coordinates are adapted to

${ }^{\mathrm{c}}$ More precisely, marginally outer-trapped surfaces. 
the physical situation. Reexpressing demands (1) and (3) in the variables of the conformal thin sandwich equations yields the following conditions:

$$
\begin{aligned}
\tilde{s}^{k} \tilde{\nabla}_{k} \ln \psi & =-\frac{1}{4} \tilde{h}^{i j} \tilde{\nabla}_{i} \tilde{s}_{j}+\frac{1}{6} \psi^{2} K-\frac{\psi^{2}}{8 N} \tilde{s}_{i} \tilde{s}_{j}(\tilde{\mathbb{L}} \beta)^{i j}, & & \text { on } \mathcal{S}, \\
\beta^{i} & =\psi^{2} N \tilde{s}^{i}+\beta_{\|}^{i}, & & \text { on } \mathcal{S},
\end{aligned}
$$

where $\tilde{s}^{k}$ is defined below Eq. (3.13), $\tilde{h}_{i j}=\tilde{g}_{i j}-\tilde{s}_{i} \tilde{s}_{j}$ denotes the induced conformal metric on $\mathcal{S}$ and $\beta_{\|}^{i}$ is the projection of $\beta^{i}$ into $\mathcal{S}$ (i.e. $\beta_{\|}^{i} \tilde{s}_{i}=0$ ). Equation (3.17) represents a nonlinear Robin-like boundary condition on $\psi$, whereas Eq. (3.18) is essentially a Dirichlet condition on the shift. The remaining demand of vanishing shear of $k^{\mu}$, finally, implies [15] that the tangential component of the shift $\beta_{\|}^{i}$ must be a conformal Killing vector of the two-dimensional surface $\mathcal{S}$,

$$
\tilde{D}^{(i} \beta_{\|}^{j)}-\frac{1}{2} \tilde{h}^{i j} \tilde{D}_{k} \beta_{\|}^{k}=0 \quad \text { on } \mathcal{S},
$$

where $\tilde{D}_{i}$ denotes the covariant derivative within $\mathcal{S}$, which is induced by the conformal metric. Cook \& Pfeiffer point out that solutions to Eq. (3.19) can be found before the conformal thin sandwich equations are solved: Each connected component of $\left(\mathcal{S}, \tilde{h}_{i j}\right)$ is topologically $S^{2}$ and therefore conformally equivalent to a unit 2 -sphere embedded in three-dimensional Euclidean space. For such a Euclidean 2sphere, Euclidean rotations around the center represent Killing vectors, which are conformal Killing vectors of any conformally related manifold, including $\left(\mathcal{S}, \tilde{h}_{i j}\right)$. Therefore, these rotations will satisfy Eq. (3.19). The freedom to specify arbitrary rotations can be used to construct black holes with arbitrary rotational state. Interestingly, the lapse boundary condition is not determined by quasi-equilibrium considerations, but is rather part of the temporal gauge choice.

The quasi-equilibrium boundary conditions at the inner excision regions allow for arbitrary specification of conformal metric, mean curvature, and shape of the excision regions. Spectral numerical solutions of the conformal thin sandwich equations (with lapse equation) using the quasi-equilibrium boundary conditions Eqs. (3.17)(3.19) were obtained in [15] for the special case of a conformally flat metric and excision of exact coordinate spheres. Three lapse boundary conditions at the excised regions (Dirichlet, von Neumann and Robin), combined with irrotational and corotating black holes, and combined with two choices for the mean curvature $K$ were explored, for a total of 12 sequences of quasi-circular orbits. Furthermore, spinning and boosted single black holes were constructed under the approximation of conformal flatness, and it was shown that the errors introduced by the use of a flat conformal metric are fairly small. Nonetheless, the freedom in the choice of $\tilde{g}_{i j}, K, \mathcal{S}$ and the lapse boundary condition can be used to further fine tune the method.

There is a close connection between the quasi-equilibrium inner boundary conditions and the isolated horizon framework developed by Ashtekar and coworkers (see, e.g. 2183]). Jaramillo et al. [26] work out this relation very clearly. Not surprisingly their results are closely related to findings in Ref. [15], including an independent argument why the lapse boundary condition is not determined by quasi- 
equilibrium/isolated horizon considerations. Ref. [26] does not present numerical results, and it is not immediately clear how to translate the conditions in this paper into a form usable in numerical simulations. Yo el. al. 47] recently solved the five coupled conformal thin sandwich equations on a Kerr-Schild background, however, with much simpler inner boundary conditions, and with much less accuracy, owing to the employed finite-difference code with uniform grid spacing.

As a final, somewhat unrelated application of the conformal thin sandwich equations, we mention construction of initial data with superposed gravitational waves 37. (see also 445]). The idea is very simple: Given a slice through a stationary background spacetime with induced metric $g_{i j}^{0}$ and mean curvature $K^{0}$, as well as a linearized gravitational wave $h_{i j}$ (either on the background, or on Minkowski space), set the free data for the conformal thin sandwich equations as

$$
\begin{aligned}
\tilde{g}_{i j} & =g_{i j}^{0}+A h_{i j}, \\
\tilde{u}_{i j} & =A \partial_{t} h_{i j}, \\
K & =K^{0}, \\
\partial_{t} K & =0,
\end{aligned}
$$

where $A$ is the adjustable amplitude of the perturbation. In Ref. 37 the conformal thin sandwich equations were solved with these free data (and Dirichlet boundary conditions). Solutions were obtained for perturbations of Minkowski space and of a Schwarzschild black hole with very large amplitudes. These perturbed black hole initial data are used to test constraint preserving boundary conditions for evolutions of Einstein's equations 28.

\section{Conclusion}

We summarized the conformal method to solve the constraints of general relativity, both in its Lagrangian and Hamiltonian viewpoints. By virtue of the recently discovered weighted transverse-traceless decomposition in the Hamiltonian viewpoint (the extrinsic curvature formulation), it is shown to be completely equivalent to the Lagrangian viewpoint (the conformal thin sandwich formulation). Both pictures are invariant to conformal transformations of the free data, and in both pictures, the decomposition of the tracefree extrinsic curvature commutes with the conformal scalings.

Subsequently, we summarized recent numerical work on solving the coupled constraint equations, in either viewpoint, concentrating on quasi-equilibrium solutions using the conformal thin sandwich equations, as well as superposed Kerr-Schild

data and initial data based on post-Newtonian results using the extrinsic curvature formulation.

\section{Acknowledgments}

I am grateful for helpful discussions with James York, Lee Lindblom and Gregory Cook. This work was supported by a Sherman Fairchild Prize fellowship and by NSF 
grants PHY-0244906 and PHY-0099568 to the California Institute of Technology.

\section{References}

[1] R. Arnowitt, S. Deser, And C. W. Misner, The dynamics of general relativity, in Gravitation: An introduction to current research, L. Witten, ed., Wiley, New York, 1962.

[2] A. Ashtekar, C. Beetle, O. Dreyer, S. Fairhurst, B. Krishnan, J. LeWAndowski, AND J. WisnieWski, Generic isolated horizons and their applications, Phys. Rev. Lett., 85 (2000), pp. 3564-3567.

[3] A. Ashtekar and B. Krishnan, Dynamical horizons and their properties, Phys. Rev. D, 68 (2003), pp. 104030/1-25.

[4] R. BAnK AND M. Holst, A new paradigm for parallel adaptive mesh refinement, SIAM Review, 45 (2003), pp. 291-323.

[5] S. Bonazzola, E. Gourgoulhon, P. Grandclément, and J. Novak, Constrained scheme for the einstein equations based on the dirac gauge and spherical coordinates, Phys. Rev. D, 70 (2004), pp. 104007-24.

[6] J. M. Bowen And J. W. York, JR., Time-asymetric initial data for black holes and black-hole collisions, Phys. Rev. D, 21 (1980), pp. 2047-2056.

[7] J. B. Boyd, Chebyshev and Fourier Spectral Methods, 2nd edition, Dover, New York, 2001. For an online version, see http://www-personal.engin.umich.edu/ jpboyd

[8] S. Brandt and B. Brügmann, A simple construction of initial data for multiple black holes, Phys. Rev. Lett., 78 (1997), pp. 3606-3609.

[9] J. D. Brown AND L. L. Lowe, Multigrid elliptic equation solver with adaptive mesh refinement, gr-qc/0411112 (2004).

[10] C. Canuto, M. Y. Hussaini, A. Quarteroni, and T. A. Zang, Spectral Methods in Fluid Dynamics, Springer, New York, 1987.

[11] G. B. Cook, Initial data for axisymmetric black-hole collisions, Phys. Rev. D, 44 (1991), pp. 2983-3000.

[12] G. B. Cook, Initial data for numerical relativity, Living Rev. Relativity, 3 (2000). http://livingreviews.org/Articles/Volume3/2000-5cook/ [Online Article]: cited on Aug 11, 2001.

[13] G. B. Cook, Corotating and irrotational binary black holes in quasicircular orbits, Phys. Rev. D, 65 (2002), p. 084003.

[14] G. B. Cook, M. W. Choptuik, M. R. Dubal, S. Klasky, R. A. Matzner, and S. R. OliveIRA, Three-dimensional initial data for the collision of two black holes, Phys. Rev. D, 47 (1993), pp. 1471-1490.

[15] G. B. Cook And H. P. PfeIfFer, Excision boundary conditions for black hole initial data, Phys. Rev. D, 70 (2004), pp. 104016-24.

[16] S. DESER, Covariant decomposition of symmetric tensors and the gravitational Cauchy problem, Ann. Inst. Henri Poincaré, Section A, 7 (1967), pp. 149-188.

[17] P. Diener, N. Jansen, A. Khokhlov, and I. Novikov, Adaptive mesh refinement approach to the construction of initial data for black hole collisions, Class. Quantum Gravit., 17 (2000), pp. 435-451.

[18] O. Dreyer, B. Krishnan, D. Shoemaker, and E. Schnetter, Introduction to isolated horizons in numerical relativity, Phys. Rev. D, 67 (2003), p. 024018.

[19] L. P. Eisenhart, Riemannian Geometry, Princeton University Press, Princeton, New Jersey, 1925.

[20] É. É. Flanagan And S. A. Hughes, Measuring gravitational waves from binary black hole coalescences. ii. the waves' information and its extraction, with and without 
templates, Phys. Rev. D, 57 (1998), pp. 4566-4587.

[21] A. Garat And R. H. Price, Nonexistence of conformally flat slices of the Kerr spacetime, Phys. Rev. D, 61 (2000), p. 124001.

[22] E. Gourgoulhon, P. Grandclément, and S. Bonazzola, Binary black holes in circular orbits. I. a global spacetime approach, Phys. Rev. D, 65 (2002), p. 044020.

[23] P. Grandclément, E. Gourgoulhon, and S. Bonazzola, Binary black holes in circular orbits. II. numerical methods and first results, Phys. Rev. D, 65 (2002), p. 044021.

[24] M. D. Hannam, C. R. Evans, G. B. Cook, And T. W. Baumgarte, Can a combination of the conformal thin-sandwich and puncture methods yield binary black hole solutions in quasi-equilibrium?, Phys. Rev. D, 68 (2003), p. 064003.

[25] M. Holst, Adaptive numerical treatment of elliptic systems on manifolds, Adv. Comput. Math., 15 (2001), pp. 139-191.

[26] J. Jaramillo, E. Gourgoulhon, And G. M. MARugÁn, Inner boundary conditions for black hole initial data derived from isolated horizons, gr-qc/0407063, (2004).

[27] P. Jaranowski AND G. SchäFer, Third post-Newtonian higher order ADM Hamilton dynamics for two-body point-mass systems, Phys. Rev. D, 57 (1998).

[28] L. E. Kidder, L. Lindblom, M. A. Scheel, L. T. Buchman, And H. P. Pfeiffer, Boundary conditions for the Einstein evolution system, in preparation, (2004).

[29] J. A. V. Kroon, Nonexistence of conformally flat slices in Kerr and other stationary spacetimes, Phys. Rev. Lett., 92 (2004), p. 041101.

[30] P. A. Lichnerowicz, Líntegration des équations de la gravitation relativiste et le problème des $n$ corps, J. Math. Pures Appl., 23 (1944), pp. 37-63.

[31] P. Marronetti and R. A. Matzner, Solving the initial value problem of two black holes, Phys. Rev. Lett., 85 (2000), p. 5500.

[32] R. A. Matzner, M. F. Huq, and D. Shoemaker, Initial data and coordinates for multiple black hole systems, Phys. Rev. D, 59 (1999), p. 024015.

[33] D. K. Monroe, Spatial conformal curvature and gravitational radiation, PhD thesis, University of North Carolina, 1976.

[34] N. Ó. Murchadha AND J. W. YORK, JR., Initial-value problem of general relativity. I. General formulation and physical interpretation, Phys. Rev. D, 10 (1974), pp. 428436.

[35] H. P. PfeIfFer, Initial Data for Black Hole Evolutions, PhD thesis, Cornell University, 2003.

[36] H. P. Pfeiffer, G. B. Cook, And S. A. Teukolsky, Comparing initial-data sets for binary black hole, Phys. Rev. D, 66 (2002), p. 024047.

[37] H. P. Pfeiffer, L. E. Kidder, M. A. Scheel, and D. Shoemaker, Initial data for Einstein's equations with superposed gravitational waves, gr-qc/0410016, (2004).

[38] H. P. Pfeiffer, L. E. Kidder, M. A. Scheel, And S. A. Teukolsky, A multidomain spectral method for solving elliptic equations, Comput. Phys. Commun., 152 (2003), pp. 253-273.

[39] H. P. Pfeiffer, S. A. Teukolsky, And G. B. Cook, Quasicircular orbits for spinning binary black holes, Phys. Rev. D, 62 (2000), p. 104018.

[40] H. P. Pfeiffer ANd J. W. York JR., Extrinsic curvature and the Einstein constraints, Phys. Rev. D, 67 (2003), p. 044022.

[41] F. Pretorius, Numerical relativity using a generalized harmonic decomposition, gr-qc/0407110 (2004).

[42] - private communication. (paper forthcoming), 2004.

[43] R. RiETH, On the validity of wilson's approach to general relativity, in Mathematics of Gravitation. Part II. Gravitational Wave Detection, A. Królak, ed., Polish Academy 
of Sciences, Institute of Mathematics, Warsaw, 1997, pp. 71-74.

[44] M. ShiBATA AND T. NAKAmuRA, Evolution of three-dimensional gravitational waves: Harmonic slicing case, Phys. Rev. D, 52 (1995), pp. 5428-5444.

[45] L. Smarr And J. W. York, JR., Radiation gauge in general relativity, Phys. Rev. D, 17 (1978), pp. 1945-1956.

[46] W. Tichy, B. Brügmann, M. Campanelli, and P. Diener, Binary black hole initial data for numerical general relativity based on post-Newtonian data, Phys. Rev. D, 67 (2003), p. 064008.

[47] H.-J. Yo, J. N. CoOk, S. L. Shapiro, And T. W. Baumgarte, Quasi-equilibrium binary black hole initial data for dynamical evolutions, gr-qc/0406020, (2004).

[48] J. W. YORK, JR., private communication.

[49] — Role of conformal three-geometry in the dynamics of gravitation, Phys. Rev. Lett., 28 (1972), pp. 1082-1085.

[50] J. W. YORK, JR., Conformally invariant orthogonal decomposition of symmetric tensors on Riemannian manifolds and the initial-value problem of general relativity, J. Math. Phys., 14 (1973), pp. 456-464.

[51] J. W. YORK, JR., Covariant decompositions of symmetric tensors in the theory of gravitation, Ann. Inst. Henri Poincaré, Section A, 21 (1974), p. 319.

[52] J. W. York, JR., Kinematics and dynamics of general relativity, in Sources of Gravitational Radiation, L. L. Smarr, ed., Cambridge University Press, Cambridge, England, 1979, p. 83.

[53] J. W. York, JR., Conformal "thin-sandwich" data for the initial-value problem of general relativity, Phys. Rev. Lett., 82 (1999), pp. 1350-1353. 